\title{
Emerging role of microRNA-27a in human malignant glioma cell survival via targeting of prohibitin
}

\author{
WEI CHEN ${ }^{1}$, JUN QI ${ }^{2}$, GANG BAO ${ }^{1}$, TUO WANG ${ }^{1}$, CHANG-WANG DU $^{1}$ and MAO-DE WANG ${ }^{1}$ \\ ${ }^{1}$ Department of Neurosurgery, The First Affiliated Hospital of Xi'an Jiaotong University, Xi'an, Shaanxi 710061; \\ ${ }^{2}$ Institute of Transfusion Research, Shaanxi Blood Center, Xi'an, Shaanxi 710061, P.R. China
}

Received May 19, 2014; Accepted February 5, 2015

DOI: $10.3892 / \mathrm{mmr} .2015 .3475$

\begin{abstract}
MicroRNAs (miRs) function as oncogenes and tumor suppressors, and have roles in most cellular processes. To date, the possible role of miR-27a, which is part of the miR-23a/27a/24-2 cluster, in malignant gliomas has remained elusive. Therefore, the present study aimed to explore the role of miR-27a in glioma and its potential target. Through transfection with miR-27a inhibitor or oligonucleotide mimics, the impact of miR-27a silencing or overexpression on the growth, apoptosis, cell cycle and invasiveness of U251 and U87MG cells was examined in vitro. The present study initially identified the potential target of miR-27a in glioma cells through a bioinformatics analysis, which was used for screening of the literature on the proteomics of gliomas. Prohibitin (PHB) was then confirmed as a target by absolute luciferase reporter assays, quantitative real-time polymerase chain reaction and western blot analysis. Treatment with miR-27a mimics oligonucleotides suppressed U251 cell proliferation, promoted apoptosis by inducing $\mathrm{G} 2 / \mathrm{M}$ phase arrest, and impaired the invasive potential of U251 cells in vitro. In addition, miR-27a expression was downregulated in glioma tissues. A PHB-3'-untranslated region luciferase reporter assay confirmed PHB as a direct target gene of miR-27a. PHB mRNA expression was reversely correlated with levels of miR-27a in U251 cells. Overexpression of miR-27a in U251 cells at $72 \mathrm{~h}$ and $96 \mathrm{~h}$ post-transfection with miR-27a mimics significantly decreased PHB protein expression by $17.2 \%$ and $43.9 \%$, respectively. In conclusion, miR-27a was shown to be a significant tumor suppressor by targeting and decreasing PHB protein expression in glioma U251 cells. miR-27a targeting of PHB may be a novel potential therapeutic strategy for glioma.
\end{abstract}

Correspondence to: Professor Mao-De Wang, Department of Neurosurgery, The First Affiliated Hospital of Xi'an Jiaotong University, 277 Yanta West Road, Xi'an, Shaanxi 710061, P.R. China E-mail: wang_maode@163.com

Key words: human malignant glioma, microRNA, U251 cells, U87MG cells, proliferation, apoptosis, invasion, prohibitin

\section{Introduction}

Malignant gliomas, the most common primary tumor type of the central nervous system, are aggressive, highly invasive, and neurologically destructive human cancers (1). Despite advances in surgical techniques, radiotherapy and chemotherapy, the prognoses of patients with malignant gliomas remain dissatisfactory (2). Establishing the molecular basis of the tumorigenesis of malignant gliomas is crucial to improving current therapies and developing novel treatments.

MicroRNAs (miRNAs or miRs) are endogenous, 19-25 nucleotides long, non-coding small RNA molecules and are emerging as important post-transcriptional regulators that either inhibit mRNA translation or direct target mRNA degradation (3). miRs are involved in tumorigenesis and tumor progression by regulating post-transcriptional gene expression. Aberrantly expressed miRs have been shown to be associated with numerous types of cancer. miRs are able to function as oncogenes as well as tumor suppressors (4). The functional mechanism and therapeutical potential of miRs specifically to gliomas are appealing, since there has been very limited progress in the development of treatments of malignant glioma in the last 25 years (5). However, studies on miRs in gliomas remain limited in number and are characterized by different or paradoxical effects among tissue specimens and studies (6).

miR-27a is part of the miR-23a/27a/24-2 cluster localized on chromosome 9q22. Several cellular targets of miR-27a are able to impact cell cycle regulation, proliferation, apoptosis and differentiation. Overexpression of miR-23a/27a/24-2 sensitized HEK293T cells to TNF- $\alpha$ cytotoxicity $(7,8)$. Data indicated that miR-27a induced increased sensitivity of leukemia (9) and JJN3 cells (multiple myeloma cell line) (10) to chemotherapy, as demonstrated by an increase the apoptotic rate. This suggested that miR-27a may be a valuable target in cancer therapy. miR-27a has been reported to be significantly upregulated in several types of human cancer (11-13), while being downregulated in others (14-16). The miR-23a/27a/24-2 cluster may exert context-dependent functions and its molecular functions are urgently required to be elucidated. Recent studies have demonstrated that miR-27a may act either as an oncogene or an anti-oncogene by targeting different downstream targets in a variety of tissues and disease states $(15,17)$. To the best of our knowledge, the possible role of miR-27a in malignant glioma has not been investigated. 
The application of proteomics to gliomas has identified lists of key proteins and signaling pathways (18). Among these important proteins, the protein prohibitin (PHB) was most commonly identified to be differentially expressed in multiple proteomic studies on glioma (19-21). Several studies demonstrated that PHB expression in human gliomas was higher than that in normal brain tissues (20-22). In fact, it is thought that PHB may have different roles in tumorigenesis, having either a permissive action on tumor growth or a tumor suppressor role $(23,24)$. Of note, PHB has a large highly conserved 3'-untranslated region (UTR) with several putative miR-binding sites, indicating that miRs may be heavily involved in its regulation. The potential targeting association between miR-27a and PHB may have an important role in the process of cancer progression, tumorigenesis or tumor suppression. To date, the association of miR-27a and PHB in gliomas has not been clarified but is worth investigating.

The present study, explored the potential role of miR-27a in malignant glioma via investigating the effects of miR-27a the cell growth, apoptosis, cell cycle and invasiveness of U251 and U87MG glioma cells in vitro. Furthermore, the targeting effect of miR-27a on PHB in glioma cells was investigated. The present study provided insight into developing novel and effective therapeutic strategies for gliomas.

\section{Materials and methods}

Cell culture and treatments. The human glioma cell line U251 and the glioblastoma cell line U87MG were obtained from the Cell Bank of Shanghai Institutes for Biological Sciences of the Chinese Academy of Sciences (Shanghai, China). The cells were cultured in DMEM (Gibco-BRL, Invitrogen Life Technologies, Carlsbad, CA, USA), supplemented with $10 \%$ fetal bovine serum (Gibco-BRL), $1.5 \mathrm{mmol} / 1 \mathrm{~L}$-glutamine, $100 \mathrm{U} / \mathrm{ml}$ penicillin and $100 \mu \mathrm{g} / \mathrm{ml}$ streptomycin in a humidified incubator containing $5 \% \mathrm{CO}_{2}$ and $95 \%$ air at $37^{\circ} \mathrm{C}$.

Oligonucleotides and cell transfection. Locked Nucleic Acid (LNA)-modified Homo sapiens (hsa)-miR-27a inhibitor (miR-27a anti-sense oligonucleotides) and mimics (miR-27a sense oligonucleotides) were chemically synthesized by Shanghai GenePharma Co., Ltd. (Shanghai, China). The negative control (NC) was a scrambled oligonucleotide that has been validated to not produce any identifiable effects on known miRNA function. The sequences of hsa-miR-27a mimics (MI), hsa-miR-27a inhibitor (IN), as well as the scrambled sequences MINC and INNC are shown in Table I. The cells were transfected with oligonucleotide using Lipofectamine 2000 reagent (Invitrogen Life Technologies) according to the manufacturer's instructions.

Real-time quantification of miR-27a and PHB mRNA by stem-loop reverse transcription quantitative polymerase chain reaction $(R T-q P C R)$. To detect the relative levels of miR-27a in glioma tissues and PHB mRNA in cell lines, RT-qPCR was performed with a universal reverse primer and miR-27a-specific primers, which were designed from the primary precursor molecule sequences of the human miR database (http://www.mirbase.org). Stem-looped reverse transcription primers of human miR-27a and PHB primers were synthesized by Shanghai GenePharma Co., Ltd. Expression of mature miR-27a was quantified by miR-qRT PCR using the Hairpin-it ${ }^{\mathrm{TM}}$ miRNAs qPCR Quantitation kit (GenePharma Co. Ltd.) and the FTC3000 Real-Time PCR detection system (Funglyn Biotech, Ontario, Canada) The results of the real-time PCR were analyzed using the $2^{-\Delta \Delta C t}$ method.

Cell proliferation. The colorimetric Cell Counting kit-8 (CCK-8) assay (Dojindo, Kumamoto, Japan) was used to measure cell proliferation. Cells transfected with $10 \mathrm{pmol}$ miR-27a mimics/inhibitor were plated at a density of 10,000 cells/well in 96-well plates. CCK- 8 solution was added to each well at $0,24,48$ and $72 \mathrm{~h}$. The optical density was then determined at $490 \mathrm{~nm}$ using a Multiskan Spectrum microplate reader (Thermo Fisher Scientific, Waltham, MA, USA).

Cell cycle analysis. After each treatment for $48 \mathrm{~h}$, nuclei of cells were stained with propidium iodide (PI; M3032; Mbchem, Shanghai, China) and examined by Cytomics FC500 Flow Cytometer (Beckman Coulter, Pasadena, CA, USA) and DNA histograms were analyzed by MultiCycle AV for windows software version 300 (Beckman Coulter).

Cell apoptosis assay. Staining with Annexin V conjugated with fluorescein isothiocyanate (FITC)/propidium iodide (PI; M3021; Mbchem, Shanghai, China) was performed according to the manufacturer's instruction and analyzed using the Cytomics FC500 Flow Cytometer (Beckman Coulter) to distinguish between live/apoptotic/necrotic cells.

Cell invasion assay. The invasive capacity of cells was determined using BD Bio Coat Matrigel invasion chambers $(8-\mu \mathrm{m}$ pores; BD Biosciences, San Jose, CA, USA). 48 h post-transfection, cells on the underside of the membrane were counted from four microscopic fields (magnification, x200; Nikon Eclipse TS100-F; Nikon, Tokyo, Japan).

Glioma samples and normal brain tissues. After informed consent from patients diagnosed with glioma was obtained, human glioma samples were collected from the Department of Neurosurgery, the First Affiliated Hospital of Xi'an Jiangtong University (Xi'an, China) in 2012. The eight glioma samples were diagnosed as World Health Organization grade II or grade III by pathological diagnosis. Four normal brain tissues were obtained following informed consent from patients with severe traumatic brain injury who required post-trauma surgery.

Databases and bioinformatics. In order to define the potential targets of miR-27a, the miRNA targets predicted by publicly available computational algorithms were obtained from miRBase Targets (http://www.ebi.ac.uk/enright-srv/microcosm/cgi-bin/targets/v5/search.pl), TargetScan (http://genes. mit.edu/tscan/targetscanS.html) and TargetScan Release 6.2 (http://www.targetscan.org/cgi-bin/vert_61/view_gene.cgi?gs $=$ PHB\&taxid $=9606 \&$ members $=\mathrm{miR}-27 \mathrm{abc} / 27 \mathrm{a}-3 \mathrm{p} \&$ showcnc $=1 \&$ shownc $=1)$ to obtain the sequences containing a seed region. Furthermore, the website http://mirecords.biolead. org/prediction_query.php was used as a multiple miRNA target prediction tool. 
Table I. Sequences of hsa-miR-27a mimics and inhibitor.

Sequences

\begin{tabular}{lll}
\cline { 2 - 3 } $\operatorname{miR}$ & \multicolumn{1}{c}{ Sense (5'-3') } & \multicolumn{1}{c}{ Antisense (5'-3') } \\
\hline hsa-miR-27a mimics & UUCACAGUGGCUAAGUUCCGC & GGAACUUAGCCACUGUGAAUU \\
Mimics NC & UUCUCCGAACGUGUCACGUTT & ACGUGACACGUUCGGAGAATT \\
hsa-miR-27a inhibitor & GCGGAACT+T+AGCCACT+GT+GAA & \\
Inhibitors NC & CAGUACUUUUGUGUAGUACAA & \\
\hline
\end{tabular}

T+: locked nucleic acid modified; hsa, Homo sapiens; NC, negative control.

Luciferase reporter assay. The full-length 3'-UTR region of the PHB gene (Gene ID, P35232) was amplified from human genomic DNA including the predicted target site for miR-27a on PHB, carrying the Xho I and Not I restriction sites. The PCR product was cloned into the multiple cloning region, located downstream of the Renilla luciferase translational stop codon in the psiCHECK-2 vector (Promega, Madison, WI, USA). 5 ng psiCHECK-2 -PHB mRNA 3'-UTR luciferase reporter vectors and 5 pmol miR-27a mimics or inhibitor or a negative control were co-transfected into U251 cells using Lipofectamine 2000 (Invitrogen Life Technologies) in 24-well plates. Cells were harvested at $48 \mathrm{~h}$ post-transfection and assayed using the Dual-Luciferase Reporter Assay System (Promega, Madison, WI, USA), according to the manufacturer's instructions. Firefly luciferase activity was normalized to Renilla luciferase activity.

Western blot analysis. 72 or $96 \mathrm{~h}$ post-transfection, U251 and U87MG cells were lysed using Mammalian Protein Extraction Reagent (78503; Thermo Fisher Scientific). The membranes were incubated at $4^{\circ} \mathrm{C}$ overnight with a mouse primary monoclonal antibody against PHB (ab55618; 1:500 dilution; Abcam, Cambridge, MA, USA) and a mouse monoclonal anti- $\beta$-actin (A5441; 1:10,000 dilution; Sigma-Aldrich, St. Louis, MO, USA), followed by incubation at room temperature for $2 \mathrm{~h}$ with horseradish peroxidase-conjugated goat anti-mouse secondary Ig antibodies (JIR 115-035-003; 1:8,000 dilution; Jackson ImmunoResearch, West Grove, PA USA). The immunoreactivity of the proteins was detected with Super Signal West Pico Chemiluminent substrates (Thermo Fisher Scientific) and exposed to X-ray film for autoradiography (Kodak, Rochester, NY, USA). The autoradiograms were scanned and analyzed with Gel-Pro analyzer software version 4.0 (Media Cybernetics, Rockville, MD USA).

Statistical analysis. Values are expressed as the mean \pm standard error of three or more experiments performed in duplicate. Statistical analysis was performed by one-way analysis of variance, repeated measures of general linear models, Fisher's least significance difference, Dunnett's t-test for multiple groups and Student's t-test for double groups using SPSS 19.0 (International Business Machines, Armonk, NY, USA). Differences from the control value were considered significant when the P-value was $<0.05$.

\section{Results}

Validation of miR-27a mimics/inhibitors in U251 and U87MG cells. After $24 \mathrm{~h}$ of transfection with miR-27a mimics, miR-27a expression increased in the MI group of U251 and U87MG cells by 5.85 - and 18.43-fold, respectively, as compared with that in the control and scramble-treated cells $[F=39.10$ $(\mathrm{P}<0.001)$ and $\mathrm{F}=51.19(\mathrm{P}<0.001)]$. At $48 \mathrm{~h}$ post-transfection, miR-27a expression was also enhanced in U251 and U87MG cells by 18.92 - and 4.37-fold, respectively $[\mathrm{F}=25.89(\mathrm{P}<0.01)$ and $\mathrm{F}=12.96(\mathrm{P}<0.01)$ for $48 \mathrm{~h}] .24 \mathrm{~h}$ post transfection with miR-27a inhibitor, miR-27a expression was suppressed in the IN groups of U251 and U87MG cells by 0.16 - and 0.10 -fold, respectively $[\mathrm{F}=16.77(\mathrm{P}<0.01)$ and $\mathrm{F}=24.85(\mathrm{P}<0.01)]$. At $48 \mathrm{~h}$ following transfection, miR-27a expression was also reduced in U251 and U87MG cells by 0.28 - and 0.29 -fold, respectively $[\mathrm{F}=5.27(\mathrm{P}<0.05)$ and $\mathrm{F}=6.52(\mathrm{P}<0.05)]($ Fig. 1).

miR-27a mimics reduce the viability of U251 cells. Compared with the control and scrambled control, transfection of U251 cells with miR-27a mimics oligonucleotides (10 pmol) impaired the proliferation of U251 cells, as indicated by the reduced viable cell number at $72 \mathrm{~h}$ post-transfection, resulting in $31.78 \%$ growth inhibition $(\mathrm{P}<0.01)$ (Fig. 2). The results indicated that miR-27a may function as a tumor suppressor in glioma U251 cells in vitro. Transfection with inhibitor oligonucleotides did not show any enhancing or significant inhibitory effects on the proliferation of U251 cells. By contrast, miR-27a had little or no effect on the proliferation of U87MG cells (data not shown).

miR-27a mimics cause cell cycle arrest in U251 cells. As shown in Fig. 3A, 48 h post-transfection with miR-27a mimics, the percentage of $\mathrm{U} 251$ cells in $\mathrm{G} 2 / \mathrm{M}$ phase was significantly increased to $50.34 \%$, compared to only 2.70 and $2.21 \%$ for the control and scramble oligonucleotides $(\mathrm{P}<0.001)$. Similarly, the G1/G0 phase populations in control and scramble-treated cells were 46.89 and $47.83 \%$, respectively. miR-27a overexpression significantly reduced the G1/G0 phase population to $14.83 \%$. These results suggested that miR-27a expression induced cell cycle arrest in $\mathrm{G} 2 / \mathrm{M}$ phase, a delay the progression of the cell cycle and inhibition of cell proliferation. However, transfection with inhibitor oligonucleotides did not affect the cell cycle in U251 cells. Little or no effect of miR-27a was found on the cell cycle of U87MG cells (data not shown). 
A

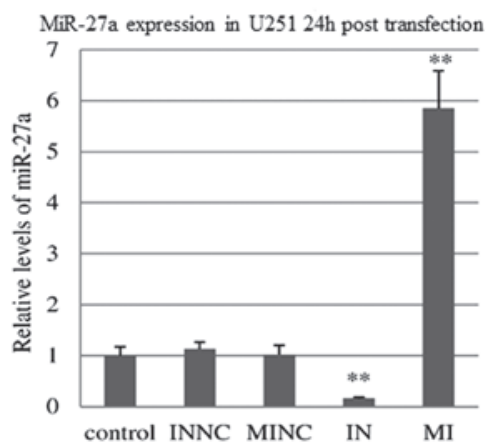

C

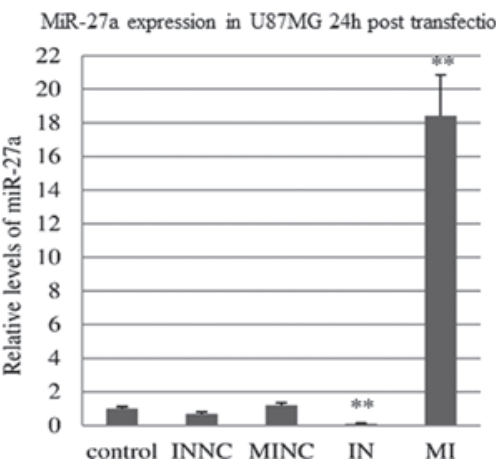

B

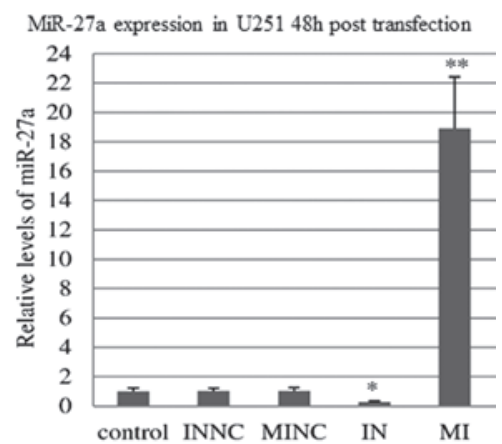

D

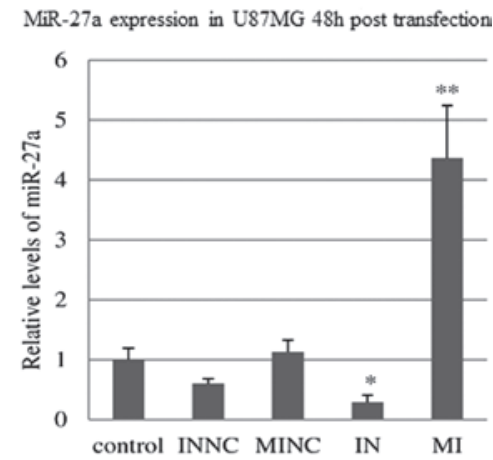

Figure 1. miR-27a was up-regulated/down-regulated via transfection with miR-27a mimics/inhibitor into U251 and U87MG cells. (A) Relative levels of miR-27a mRNA expression in U251 cells were measured $24 \mathrm{~h}$ post-transfection with MI/IN or MINC/INNC. (B) Relative levels of miR-27a mRNA expression in U251 cells were measured $48 \mathrm{~h}$ post-transfection with MI, IN, MINC or INNC. (C) Relative levels of miR-27a mRNA expression in U87MG cells were measured $24 \mathrm{~h}$ post-transfection with MI, IN, MINC or INNC. (D) Relative levels of miR-27a mRNA expression in U87MG cells were measured $48 \mathrm{~h}$ post-transfection with MI, IN, MINC or INNC. Error bars represent the standard error of triplicate independent experiments. Statistical analyses were performed using one-way analysis of variance, Fisher's least significant difference and Dunnett's t-test. ${ }^{*} \mathrm{P}<0.05,{ }^{* * *} \mathrm{P}<0.01$ compared with control and NC groups. MI, miR-27a mimics; IN, miR-27a inhibitor; INNC, scrambled inhibitor control; MINC, scrambled mimic control.

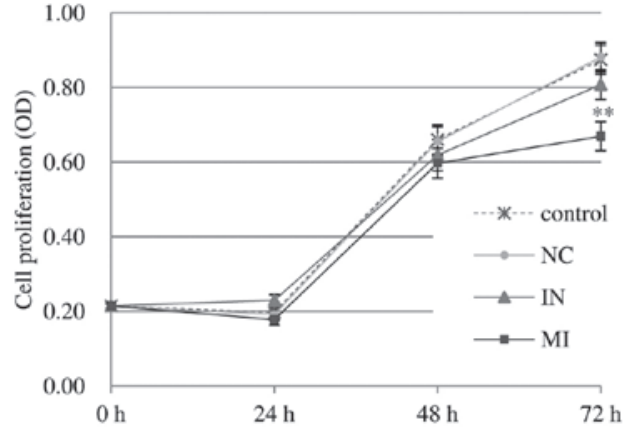

Figure 2. Proliferation of U251 cells three days following miR-27a mimics transfection. Compared with the control and NC, significant proliferation inhibition was observed at $72 \mathrm{~h}$ post-transfection $(\mathrm{P}<0.05)$. Error bars represent the standard error obtained from three independent experiments. Statistical analyses were performed using repeated measures of general linear models of analysis of variance. ${ }^{* *} \mathrm{P}<0.01$ compared with control and NC groups. MI, miR-27a mimics; IN, miR-27a inhibitor; NC, scrambled control; OD, optical density.

miR-27a mimics promote apoptosis in U251 cells. Following $48 \mathrm{~h}$ of tranfection of miR-27a mimics, flow cytometric analysis revealed that $8.47 \%$ of U251 cells were in the lower right (LR) quadrant (Annexin $\mathrm{V}^{+} / \mathrm{PI}^{-}$; early apoptotic cells) of the PI vs. Annexin V dot plot compared with only $0.26,0.44$ and $0.54 \%(\mathrm{P}<0.001)$ in the inhibitor, scramble oligonucleotides and control groups in the LR quadrant, respectively.
Furthermore, $0.57 \%$ of cells were in the upper right (UR) quadrant (Annexin $\mathrm{V}^{+} / \mathrm{PI}^{+}$; late apoptotic cells) compared to only $0.16,0.33$ and $0.24 \%(\mathrm{P}<0.05)$ in the inhibitor, scramble oligonucleotides and control groups in the UR quadrant, respectively (Fig. 3B). The significantly increased number of early apoptotic and late apoptotic cells observed in U251 cells confirmed that miR-27a inhibited glioma cell growth predominantly via inducing early apoptosis. miR-27a inhibitor transfection decreased the apoptotic rate of U251 cells, but without statistical significance. Little or no differences were found in the apoptotic rate of U87MG cell groups (data not shown).

miR-27a mimics impair the invasive ability of U251 glioma cells. The number of U251 cells invading through the Matrigel following miR-27a mimics transfection was $94.20 \pm 10.60$ cells, which was half of that in the blank control $(206.00 \pm 10.10)$ and

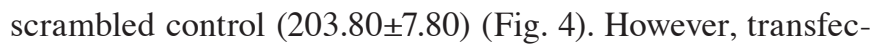
tion with miR-27a inhibitor didn't affect the invasiveness of U251 cells. Little or no differences were found in cell invasiveness of U87MG cells (data not shown).

miR-27a has low expression in glioma samples. As the effects of miR-27a observed in U251 cells were not present in U87MG cells, the present study assessed miR-27a expression in glioma samples diagnosed as grade II and III, but not grade IV. The results showed that the relative expression levels of miR-27a 
A

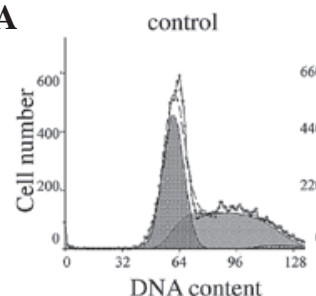

IN

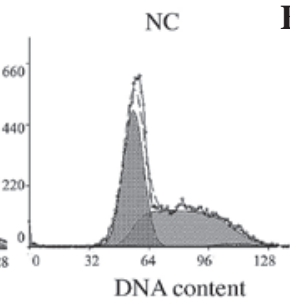

MI

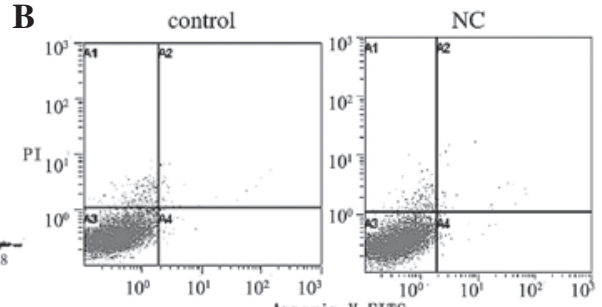

MI
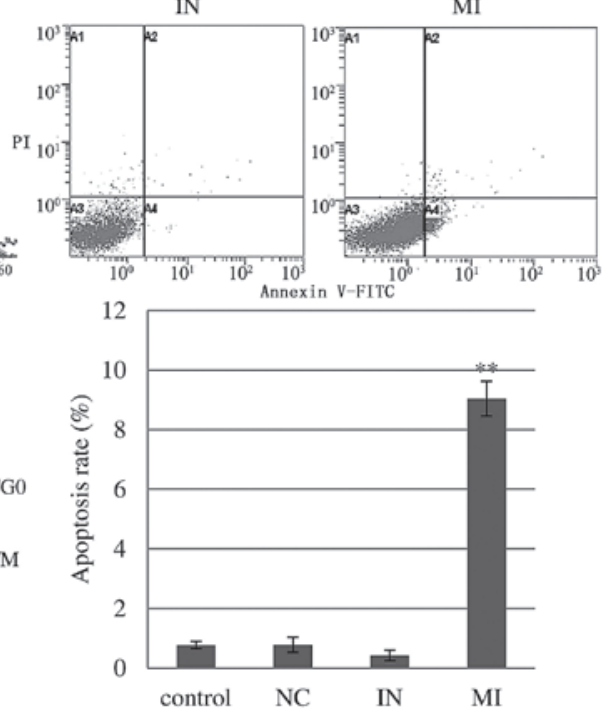

Figure 3. Effect of miR-27a on cell cycle and apoptosis. (A) Overexpression of miR-27a induced G2/M phase arrest in U251 cells. Cell cycle analysis indicated the differential percentages of cells transfected with control, NC, IN or MI in G1/G0, S and G2/M phases, respectively. Flow cytometry data (mean values) are presented as histograms and reveal a significant increase in the percentage of cells in G2/M phase in the MI group ( $\mathrm{P}<0.001)$, accompanied with a significant decrease in the G1/G0 population. (B) Apoptosis of U251 cells transfected with miR-27a mimics/inhibitor $48 \mathrm{~h}$ post-transfection. miR-27a mimics significantly elevated the early apoptotic rate. Bar graphs present the percentage of apoptotic cells (Annexin $\mathrm{V}^{+}$) calculated from each group. Error bars represent the standard deviation obtained from three independent experiments. Statistical analyses were performed using analysis of variance, Fisher's least significant difference and Dunnett's t-test. ${ }^{* *} \mathrm{P}<0.01$ compared to control and NC groups. MI, miR-27a mimics; IN, miR-27a inhibitor; NC, scrambled control.

A
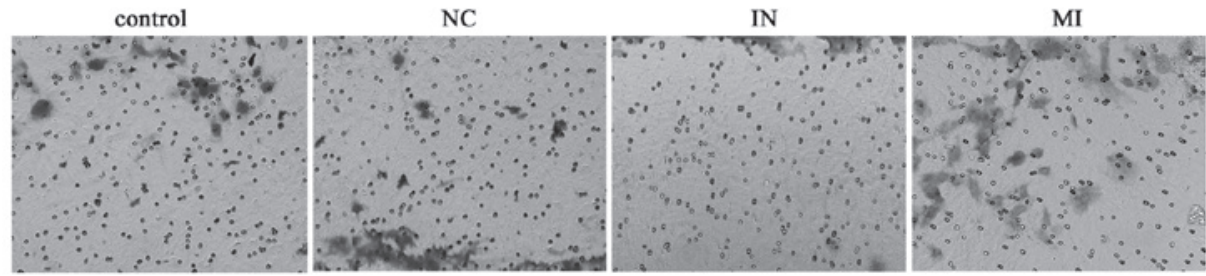

B

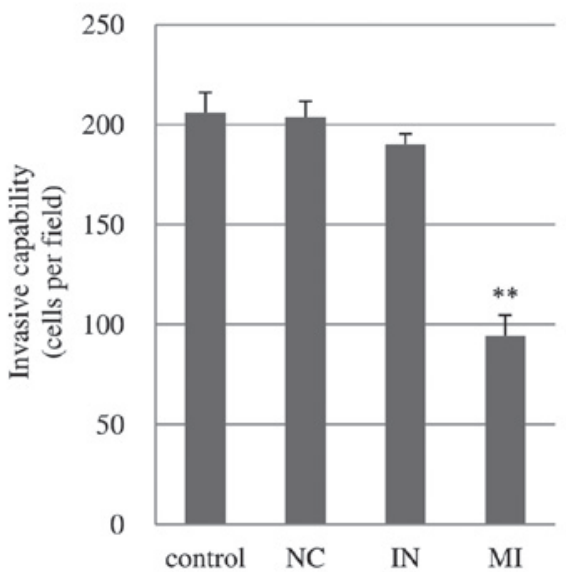

Figure 4. (A) miR-27a impaired U251 glioma cell invasiveness after 48 h of incubation (crystal violet staining; magnification, x10). (B) Quantification of the number of cells invading through the Matrigel to the lower surface of the polycarbonic membrane. The invasive potential of U251 cells transfected with miR-27 mimics was reduced by $54 \%(\mathrm{P}<0.001)$ compared to that of the control, whereas no difference was observed in U251 cells transfected with miR-27a inhibitor $(\mathrm{P}=0.213)$. Error bars represent the standard error obtained from three independent experiments. Statistical analyses were performed using one-way analysis of variance, Fisher's least significant difference and Dunnett's t-test. ${ }^{* *} \mathrm{P}<0.01$ compared to control and NC groups. MI, miR-27a mimics; IN, miR-27a inhibitor; NC, scrambled control. 


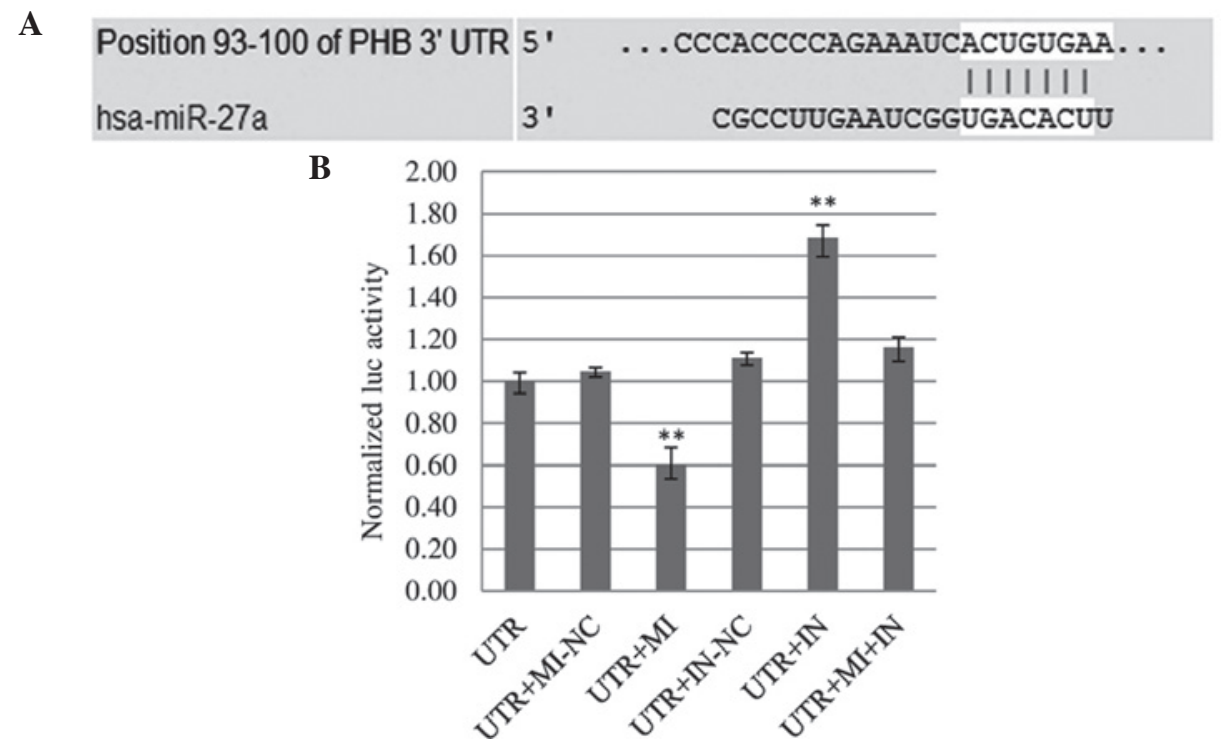

Figure 5. Effects of the predicted miR-27a target site derived from the PHB 3'-UTR on luciferease expression. (A) The alignment between the predicted miR-27a target site and binding sequence in the PHB 3'-UTR predicted by TargetScan Release 62. (B) The psiCHECK-2 -PHB mRNA 3'-UTR luciferase reporter vector was co-transfected into U251 cells along with a MI/IN or their respective NC vectors. The ratio of normalized sensor to control luciferase activity is shown. The results demonstrated that miR-27a regulated the expression of PHB mRNA through the predicted target site. Error bars represent the standard deviation obtained from three independent experiments. Statistical analyses were performed using one-way analysis of variance, Fisher's least significant difference and Dunnett's t-test. * $\mathrm{P}<0.01$ compared to the control group. hsa, Homo sapiens; UTR, 3'-untranslated region luciferase reporter vector; PHB, prohibitin; MI, miR-27a mimics; IN, miR-27a inhibitor; NC, scrambled control.

in the glioma group were significantly lower than those in the normal brain group $(0.35 \pm 0.05$ vs. $1.05 \pm 0.19 ; \mathrm{P}<0.01)$.

miR-27a directly regulates the expression of PHB. To explore the mechanism of action of miR-27a in glioma, online miRNA target prediction tools were used for bioinformatics analysis, which predicted a potential interaction between miR-27a and PHB. Using Target Scan Release 6.2, alignment between the predicted miR-27a target site and binding sequence in the PHB 3'-UTR was obtained (Fig. 5A). Absolute luciferase reporter assays demonstrated that $48 \mathrm{~h}$ post-co-transfection, overexpression of miR-27a (UTR+MI group) produced an obvious reduction (42.54\%) in luciferase expression in U251 cells as compared with the control (UTR+MI-NC) (Fig. 5B). This indicated that miR-27a mimics bound to the PHB mRNA 3'-UTR and inhibited the expression of renilla. Furthermore, downregulation of miR-27a (UTR+IN group) resulted in a significant increase $(33.90 \%)$ in luciferase activity. When miR-27a mimics and inhibitor were transfected into U251 cells simultaneously (UTR+MI+IN group), luciferase activity was higher than that in the UTR group, but lower than that in the UTR+IN group. This indicated that the miR-27a inhibitor bound not only intrinsical miR-27a in cells but also exogenous transfected miR-27a mimics. The luciferase assay confirmed that miR-27a directly regulates PHB in U251 cells by binding to the predicted target site in the 3'-UTR of PHB mRNA.

PHB mRNA expression is negatively correlated with miR-27a levels in U251 cells. When miR-27a was silenced by miR-27a inhibitor for 24 and $48 \mathrm{~h}$ in U251 cells, PHB mRNA was subsequently elevated by 36.71 and $83.87 \%$, respectively, compared with the scrambled control (Fig. 6A). PHB mRNA expression in U251 cells was decreased by 48.08 and $43.96 \%$ following transfection with miR-27a mimics for 24 and $48 \mathrm{~h}$, respectively (Fig. 6A). The results indicated that miR-27a regulates endogenous PHB mRNA levels through a mechanism of mRNA degradation. Similarly, in U87MG cells, miR-27a inhibitor/mimics were able to up-regulate/down-regulate PHB mRNA expression $24 \mathrm{~h}$ post-transfection (Fig. 6A). However, there was no correlation between miR-27a expression and PHB mRNA expression in U87MG cells $48 \mathrm{~h}$ post-transfection with miR-27a inhibitor/mimics (data not shown).

miR-27a negatively regulates $P H B$ protein expression in $U 251$ cells. Overexpression of miR-27a in U251 cells 72 and 96 h post-transfection with miR-27a mimics significantly decreased PHB protein expression levels by 17.18 and $43.90 \%(\mathrm{P}<0.01)$, respectively (Fig. 6B). Silencing of miR-27a expression in U251 cells didn't increase PHB protein expression. In U87MG cells, there was no correlation between miR-27a expression and PHB protein expression at 72 or $96 \mathrm{~h}$ post-transfection (data not shown). Similarly, transfection with miR-27a mimics or inhibitor for $48 \mathrm{~h}$ did not affect the levels of PHB mRNA in U87MG cells. This may be the reason why up- or downregulation of miR-27a was not able to affect PHB protein expression.

\section{Discussion}

MiR-27a was shown to impact cell cycle regulation, proliferation, apoptosis and invasiveness via regulating post-transcriptional target genes. Previous studies showed that miR-27a is significantly upregulated in several types of human cancer, including breast (12), kidney (11), hepatocellular (13) and gastric (25) cancer. Increased expression of miR-27a was shown to initiate tumor development by targeting certain cellular factors, including FOXO-1 (11), Myt-1 (26), ZBTB10/RINZF (a putative zinc finger and BTB domain-containing protein) $(26,27)$ and RYBP/DEDA (an 
A

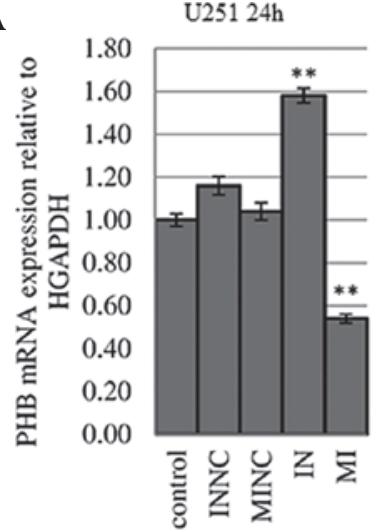

$\mathrm{U} 25148 \mathrm{~h}$

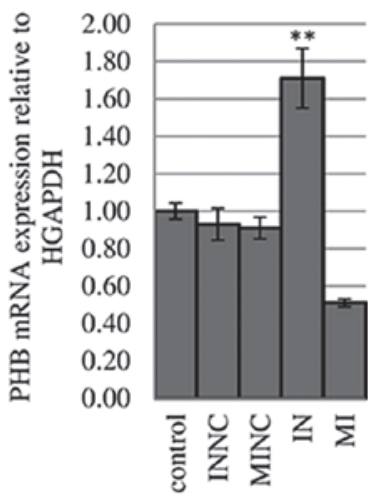

U87MG 24h

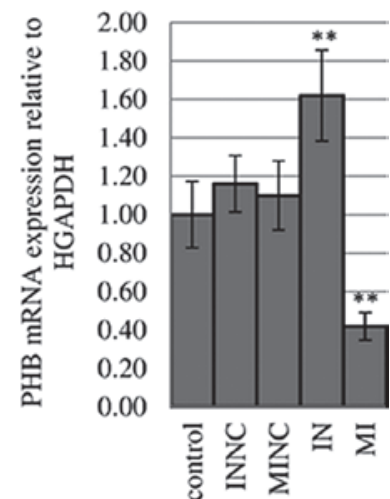

B $72 \mathrm{~h}$

$96 \mathrm{~h}$

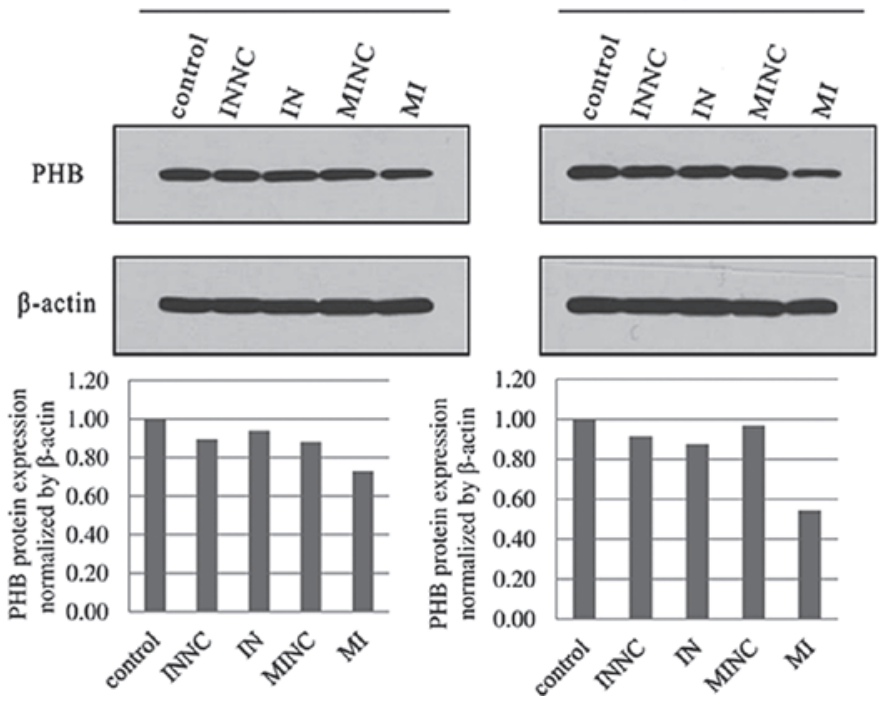

Figure 6. Effect of miR-27a on PHB expression in U251 cells. (A) Expression of PHB mRNA in U251 and U87MG cells regulated by miR-27a inhibitor/mimics PHB mRNA expression was negatively correlated with levels of miR-27a in U251 cells. (B) Western blot analysis was performed 72 and $96 \mathrm{~h}$ post-transfection with miR-27a inhibitor/mimics. Compared with the control and negative control group, overexpression of miR-27a in U251 cells downregulated PHB at the translational level. Error bars represent the standard error obtained from three independent experiments. Statistical analyses were performed using one-way analysis of variance, Fisher's least significant difference and Dunnett's t-test. ${ }^{* *} \mathrm{P}<0.01$ compared with the control group. PHB, prohibitin; MI, miR-27a mimics; IN, miR-27a inhibitor; NC, scrambled control.

apoptotic facilitator) (27). miR-27a is upregulated in pediatric B-cell acute lymphoblasic leukemia and acts as a suppressor of the tumor suppressor FBW7. FBW7 acts as a negative regulator of proliferation by facilitating proteasome degradation of cyclin E, c-Myc, c-Jun and Notch 1 (28). The experimental studies which reported the abovementioned findings support the oncogenic role of miR-27a in breast $(12,26,27)$, gastric (29), colon (30) and prostate cancer cells. However, in certain types of cancer, including acute promyelocytic leukemia (15), colorectal cancer $(16,31)$, malignant melanoma (32), oral squamous cell carcinoma (33) and prostate cancer (14), expression of miR-27a was shown to be downregulated. In the present study, miR-27a expression was significantly downregulated in glioma tissue diagnosed as grade II-III as compared with that in normal brain tissue. This indicated that miR-27a is dysregulated in human glioma and may have a role in tumorigenesis. The number of normal and tumor samples used in the present study is not large enough to statistically validate whether miR-27a expression levels are correlated with the pathological grade and malignancy of glioma, and therefore, further studies using an expanded sample size are required.
Through a series of in vitro assays in the present study, miR-27a was shown to have significant anti-proliferative effect. The significantly increased number of early and late apoptotic cells observed in U251 cells confirmed that miR-27a inhibited glioma cell growth via inducing apoptosis, as well as arresting the progression of the cell cycle and arising from a block or delay in the G2/M phase. Furthermore, miR-27a overexpression impaired the invasive potential of U251 glioma cells in vitro. All these results indicated that miR-27a may function as a tumor suppressor in glioma U251 cells. In analogy with the results of the present study, miR-27a has also been reported to have anti-tumor activity by certain studies $(9,15,17)$. Scheibner et al $(34)$ reported that miR-27a exerted its tumor suppressor-like activity in acute leukemia cells via regulation of apoptosis. Additionally, overexpression of the miR-23a/27a/24-2 cluster promoted caspase-dependent and -independent apoptosis in human embryonic kidney cells, as well as sensitized HEK293T cells to TNF-alpha cytotoxicity (7). Recently, Wang et al (17) reported that induction of miR-27a strongly decreased the proliferation of human lung cancer H1299 cells and breast cancer MDA-MB-468 cells 
in vitro as well as tumor formation in vivo. These results indicated that miR-27a may act either as an oncogene or an anti-oncogene by targeting various cellular proteins in a number of tissues and disease stages.

The siCHECK ${ }^{\mathrm{TM}}$ vectors were designed to provide a quantitative and rapid approach for identification of the target genes of miR. These vectors enable the monitoring of changes in expression of a target gene fused to the reporter gene Renilla luciferase. The present study showed that miR-27a was able to directly bind to the 3'-UTR of the PHB gene and induce cleavage of PHB mRNA, resulting in the loss-of-function of PHB. PHB is an evolutionarily conserved gene that is ubiquitously expressed and is localized to the mitochondria, the nucleus and the plasma membrane (35). The least controversial and best-characterized function of the mitochondrial PHB is as chaperones involved in the stabilization and maintenance of mitochondrial function and protection against senescence (36). The downregulation of PHB through targeting by miR-27a mimics may have changed the aberrant mitochondrial morphology and functions, which increased the sensitivity of U251 cells to apoptotic stimuli. Several studies have supported the notion that knockdown of PHB facilitates cellular apoptosis via fragmentation of the mitochondrial network $(37,38)$. There is controversy among studies in regard to the function of PHB in cellular differentiation and apoptosis $(39,40)$. It is associated with the differential phosphorylation, intracellular concentration of PHB, the cell type and the tumor stage. Merkwirth et al (39) demonstrated that prohibitins exert their anti-apoptotic function via OPA1, a dynamin-like guanin triphosphatase. Recent findings demonstrated an unexpected indispensable role of PHB in the activation of the Raf/mitogen-activated protein kinase kinase (MEK)/extracellular signal-regulated kinase (ERK)1/2 pathway via active Ras as well as in modulating epithelial cell adhesion and migration $(37,41)$. Silencing of PHB expression by miR-27a mimics sensitized U251 to apoptosis and produced a negative impact on cellular invasion via inhibition of the Ras-Raf-MEK-ERK pathway. Studies performed on cancer cell lines further demonstrated that the levels of phospho-PHB in the plasma membrane were correlated with the invasiveness of human cancer cells (42). Additionally, several studies demonstrated that the overexpression of PHB inhibited cytochrome $c$ release by decreasing the mitochondrial membrane potential and decreasing the level of B-cell lymphoma 2 (Bcl-2) (37,43). Downregulation of PHB by miR-27a mimics resulted in inhibition of the $\mathrm{Bcl}-2 / \mathrm{Bcl}-2$ extra large protein pathway and enhancement of $\mathrm{Bcl}-2$-associated $\mathrm{X}$ protein-Bcl-2 homologous antagonist killer directly by increasing cytochrome $\mathrm{C}$ from the intermitochondrial space, leading to the induction of downstream activation of cleaved caspase 3 (37). Furthermore, studies showed that PHB inhibited apoptosis by downregulating E2F activity when $\mathrm{Rb}$ family members were inactive (44). It is therefore indicated that downregulation of PHB in U251 mediated by miR-27a mimics induced apoptosis attributing to the release of free E2F1, which has been shown to promote apoptosis in several experimental systems reviewed in Phillips and Vousden (45). In addition, U251 is a p53-mutant glioma cell line. The dysregulated expression of PHB was previously reported in multiple proteomic studies on glioma (20-22). Therefore, in glioma cells, PHB was not able to function as a regulator at a physiological dose which keeps the balance between cell hyper-proliferation and apoptosis through regulating the p53 signaling pathway (46). Studies performed to date have suggested that PHB functions as a 'molecular switch', which determines cell fate. Targeting PHB may therefore be a useful therapeutic approach for the treatment of gliomas.

Compared with the MI group, silencing of miR-27a did not result in any effects opposite of those of miR-27a upregulation on cellular proliferation, apoptosis, cell cycle and invasiveness in the present study. The present study demonstrated that, in U251 cells, upregulation of miR-27a expression by mimics not only induced PHB mRNA degradation, but also inhibited its translation. miR-27a inhibition ultimately didn't lead to any alterations in PHB protein expression, although PHB mRNA expression was negatively correlated with miR-27a levels. This implied that decreasing PHB protein expression was a key factor in the anti-oncogenic role of miR-27a. However, miR-27a can be a downstream transcriptional target of certain oncogenes, which bind to the miR-27a promoter region and suppress its expression (17). These two differential pathways can have an anti-tumorigenic role via overexpression of miR-27a. The results of the present study implied that upregulation of miR-27a may exert a distinct anti-tumorigenic effect. The glioblastoma cell line U87MG with mutant PTEN is classified as grade IV (47), possessing higher malignancy and complexity in tumorigenesis than U251 cells (http://www.atcc. org/products/all/HTB-14.aspx;http:/www.sigmaaldrich.com/ catalog/product/sigma/09063001?lang=zh\&region=CN;http:// www.cellbank.org.cn/detail_1.asp?id=137\&serial=TCHu\%20 58). Alterations in miR-27a expression only had a minor influence on PHB mRNA and protein in U87MG cells. It was difficult to disturb U87MG cellular processes via transfection with miR-27a mimics or inhibitor.

In conclusion, miR-27a was downregulated in glioma diagnosed as grade II-III and acted as a tumor suppressor, which inhibits proliferation and invasion of glioma U251 cells via inducing apoptosis and cell cycle arrest. PHB is an endogenous target of miR-27a in gliomas. Overexpression of miR-27a was shown to have a significant tumor suppressive action by targeting and decreasing PHB protein expression. miR-27a targeting of PHB therefore has the potential to become a novel therapeutic strategy for human maglinant glioma.

\section{References}

1. Wen PY and Kesari S: Malignant gliomas in adults. N Engl J Med 359: 492-507, 2008.

2. Gabayan AJ, Green SB, Sanan A, et al: GliaSite brachytherapy for treatment of recurrent malignant gliomas: a retrospective multi-institutional analysis. Neurosurgery 58: 701-709, 2006.

3. Bartel DP: MicroRNAs: target recognition and regulatory functions. Cell 136: 215-233, 2009.

4. Cho WC: OncomiRs: the discovery and progress of microRNAs in cancers. Mol Cancer 6: 60, 2007.

5. Anderson E, Grant R, Lewis SC and Whittle IR: Randomized Phase III controlled trials of therapy in malignant glioma: where are we after 40 years? Br J Neurosurg 22: 339-349, 2008.

6. Auffinger B, Thaci B, Ahmed A, et al: MicroRNA targeting as a therapeutic strategy against glioma. Curr Mol Med 13: 535-542, 2013.

7. Chhabra R, Adlakha YK, Hariharan M, Scaria V and Saini N: Upregulation of miR-23a-27a-24-2 cluster induces caspase-dependent and -independent apoptosis in human embryonic kidney cells. PLoS One 4: e5848, 2009. 
8. Chhabra R, Dubey R and Saini N: Cooperative and individualistic functions of the microRNAs in the miR-23a 27a 24-2 cluster and its implication in human diseases. Mol Cancer 9: 232, 2010.

9. Feng DD, Zhang H, Zhang P, et al: Down-regulated miR-331-5p and miR-27a are associated with chemotherapy resistance and relapse in leukaemia. J Cell Mol Med 15: 2164-2175, 2011.

10. Ballabio E, Armesto M, Breeze CE, et al: Bortezomib action in multiple myeloma: microRNA-mediated synergy (and miR-27a/CDK5 driven sensitivity)? Blood Cancer J 2: e83, 2012.

11. Chow TF, Youssef YM, Lianidou E, et al: Differential expression profiling of microRNAs and their potential involvement in renal cell carcinoma pathogenesis. Clin Biochem 43: 150-158, 2010.

12. Guttilla IK and White BA: Coordinate regulation of FOXO1 by miR-27a, miR-96 and miR-182 in breast cancer cells. J Bio Chem 284: 23204-23216, 2009.

13. Huang S, He X, Ding J, et al: Upregulation of miR-23a approximately 27 a approximately 24 decreases transforming growth factor-beta-induced tumor-suppressive activities in human hepatocellular carcinoma cells. Int J Cancer 123: 972-978, 2008.

14. Prueitt RL, Yi M, Hudson RS, et al: Expression of microRNAs and protein-coding genes associated with perineural invasion in prostate cancer. Prostate 68: 1152-1164, 2008.

15. Saumet A, Vetter G, Bouttier M, et al: Transcriptional repression of microRNA genes by PML-RARA increases expression of key cancer proteins in acute promyelocytic leukemia. Blood 113 412-421, 2009

16. Xi Y, Shalgi R, Fodstad O, Pilpel Y and Ju J: Differentially regulated micro-RNAs and actively translated messenger RNA transcripts by tumor suppressor p53 in colon cancer. Clin Cancer Res 12 (7 Pt 1): 2014-2024, 2006.

17. Wang W, Cheng B, Miao L, Mei Y and Wu M: Mutant p53-R273H gains new function in sustained activation of EGFR signaling via suppressing miR-27a expression. Cell Death Dis 4: e574, 2013.

18. Deighton RF, McGregor R, Kemp J, McCulloch J and Whittle IR: Glioma pathophysiology: insights emerging from proteomics. Brain Pathol 20: 691-703, 2010.

19. Chumbalkar VC, Subhashini C, Dhople VM, et al: Differential protein expression in human gliomas and molecular insights. Proteomics 5: 1167-1177, 2005.

20. Hiratsuka M, Inoue T, Toda T, et al: Proteomics-based identification of differentially expressed genes in human gliomas: down-regulation of SIRT2 gene. Biochem Biophys Res Commun 309: 558-566, 2003.

21. Iwadate Y, Sakaida T, Hiwasa T, et al: Molecular classification and survival prediction in human gliomas based on proteome analysis. Cancer Res 64: 2496-2501, 2004.

22. Zhou JQ, Wang JT, Liu QH, Guo Xb, Zhou J and Song LJ Proteomic profiling and identification of malignant grade related proteins in human brain astrocytoma. Chin J Neuromed 11: 780-783, 2012

23. Mishra S, Murphy LC and Murphy LJ: The Prohibitins: emerging roles in diverse functions. J Cell Mol Med 10: 353-363, 2006.

24. Mishra S, Murphy LC, Nyomba BL and Murphy LJ: Prohibitin: a potential target for new therapeutics. Trends Mol Med 11: 192-197, 2005.

25. Liu T, Tang H, Lang Y, Liu M and Li X: MicroRNA-27a functions as an oncogene in gastric adenocarcinoma by targeting prohibitin. Cancer Lett 273: 233-242, 2009.

26. Mertens-Talcott SU, Chintharlapalli S, Li X and Safe S: The oncogenic microRNA-27a targets genes that regulate specificity protein transcription factors and the G2-M checkpoint in MDA-MB-231 breast cancer cells. Cancer Res 67: 11001-11011, 2007.

27. Scott GK, Mattie MD, Berger CE, Benz SC and Benz CC: Rapid alteration of microRNA levels by histone deacetylase inhibition. Cancer Res 66: 1277-1281, 2006.
28. Lerner M, Lundgren J, Akhoondi S, et al: MiRNA-27a controls FBW7/hCDC4-dependent cyclin E degradation and cell cycle progression. Cell Cycle 10: 2172-2183, 2011.

29. Belair C, Darfeuille F and Staedel C: Helicobacter pylori and gastric cancer: possible role of microRNAs in this intimate relationship. Clin Microbiol Infect 15: 806-812, 2009.

30. Chintharlapalli S, Papineni S, Abdelrahim M, et al: Oncogenic microRNA-27a is a target for anticancer agent methyl 2-cyano3,11-dioxo-18beta-olean-1,12-dien-30-oate in colon cancer cells. Int J Cancer 125: 1965-1974, 2009.

31. Volinia S, Calin GA, Liu CG, et al: A microRNA expression signature of human solid tumors defines cancer gene targets. Proc Natl Acad Sci USA 103: 2257-2261, 2006.

32. Schultz J, Lorenz P, Gross G, Ibrahim S and Kunz M: MicroRNA let-7b targets important cell cycle molecules in malignant melanoma cells and interferes with anchorage-independent growth. Cell Res 18: 549-557, 2008.

33. Kozaki K, Imoto I, Mogi S, Omura K and Inazawa J: Exploration of tumor-suppressive microRNAs silenced by DNA hypermethylation in oral cancer. Cancer Res 68: 2094-2105, 2008.

34. Scheibner KA, Teaboldt B, Hauer MC, et al: MiR-27a functions as a tumor suppressor in acute leukemia by regulating 14-3-3theta. PLoS One 7: e50895, 2012.

35. Zhou TB and Qin YH: Signaling pathways of prohibitin and its role in diseases. J Recept Signal Transduct Res 33: 28-36, 2013.

36. Nijtmans LG, de Jong L, Artal Sanz M, et al: Prohibitins act as a membrane-bound chaperone for the stabilization of mitochondrial proteins. EMBO J 19: 2444-2451, 2000.

37. Chowdhury I, Thompson WE, Welch C, Thomas $\mathrm{K}$ and Matthews R: Prohibitin (PHB) inhibits apoptosis in rat granulosa cells (GCs) through the extracellular signal-regulated kinase 1/2 $($ ERK $1 / 2)$ and the Bcl family of proteins. Apoptosis 18: $1513-1525,2013$

38. Kasashima K, Ohta E, Kagawa Y and Endo H: Mitochondrial functions and estrogen receptor-dependent nuclear translocation of pleiotropic human prohibitin 2. J Biol Chem 281: 36401-36410, 2006.

39. Merkwirth C, Dargazanli S, Tatsuta T, et al: Prohibitins control cell proliferation and apoptosis by regulating OPA1-dependent cristae morphogenesis in mitochondria. Genes Dev 22: 476-488, 2008.

40. Ross JA, Nagy ZS and Kirken RA: The PHB1/2 phosphocomplex is required for mitochondrial homeostasis and survival of human T cells. J Biol Chem 283: 4699-4713, 2008

41. Rajalingam K, Wunder C, Brinkmann V, et al: Prohibitin is required for Ras-induced Raf-MEK-ERK activation and epithelial cell migration. Nat Cell Biol 7: 837-843, 2005.

42. Chiu CF, Ho MY, Peng JM, et al: Raf activation by Ras and promotion of cellular metastasis require phosphorylation of prohibitin in the raft domain of the plasma membrane. Oncogene 32: 777-787, 2013.

43. Muraguchi T, Kawawa A and Kubota S: Prohibitin protects against hypoxia-induced $\mathrm{H} 9 \mathrm{c} 2$ cardiomyocyte cell death. Biomed Res 31: 113-122, 2010.

44. Fusaro G, Wang S and Chellappan S: Differential regulation of $\mathrm{Rb}$ family proteins and prohibitin during camptothecin-induced apoptosis. Oncogene 21: 4539-4548, 2002.

45. Phillips AC and Vousden KH: E2F-1 induced apoptosis. Apoptosis 6: 173-182, 2001

46. Fusaro G, Dasgupta P, Rastogi S, Joshi B and Chellappan S: Prohibitin induces the transcriptional activity of p53 and is exported from the nucleus upon apoptotic signaling. J Biol Chem 278: 47853-47861, 2003.

47. Clark MJ, Homer N, O'Connor BD, et al: U87MG decoded: the genomic sequence of a cytogenetically aberrant human cancer cell line. PLoS Genet 6: e1000832, 2010. 